\title{
Análise espacial da produtividade do café na região Sudeste do Brasil: 1990-2015
}

\author{
Daniela Peres Cardozo ${ }^{1}$ \\ Luana Las Schaab \\ José Luiz Parre
}

\begin{abstract}
Resumo: O objetivo deste artigo é analisar a evolução da produtividade média do setor cafeeiro da região sudeste nos anos de quinquênio de 1990 a 2015. Para tanto, analisouse a dinâmica espacial, a formação de clusters e, por fim, verificou-se a incidência de convergência na produtividade média do café. A metodologia utilizada é a análise exploratória de dados espaciais e a econometria espacial. Os resultados indicam que existe autocorrelação espacial global positiva. Houve convergência em todos os subperíodos analisados, dado que o valor do coeficiente estimado foi negativo e significativo. Assim, os resultados corroboram a hipótese de convergência espacial, indicando que a produção de café nas microrregiões menos produtivas cresceu a uma taxa maior do que nas microrregiões mais produtivas.

Palavras-chave: Convergência. Econometria-espacial. Café.
\end{abstract}

Spatial analysis of coffee productivity in the southeast region of Brazil: 1990-2015

\begin{abstract}
The objective of this article is to analyze the evolution of the average productivity of the coffee sector of the Southeast region in the five - year period from 1990 to 2015. In order to do so, it analyzed the spatial dynamics, the formation of clusters and, finally, the incidence of convergence in the average productivity was verified. The methodology used was the exploratory spatial data analysis (AEDE) and spatial econometrics. The average productivity was measured by the quantity produced (in kilograms) per planted area (in hectares). The results indicate that there is positive global spatial autocorrelation. There was convergence in all analyzed subperiods, since the value of the coefficient $\beta$ was negative and significant. So, corroborate the hypothesis of spatial convergence, indicating that the production of the coffee in less productive micro-regions grew at a higher rate than at the more productive micro-regions.
\end{abstract}

Keywords: Convergence. Spatial Econometric. Coffee.

Classificação JEL: C31, R11

1 Mestra em Ciências Econômicas pela Universidade Estadual de Maringá (UEM). E-mail: daniyellaperes@hotmail.com.

2 Mestra em Ciências Econômicas pela Universidade Estadual de Maringá (UEM). E-mail: luanalasscaab@gmail.com.

3 Professor titular do Departamento de Economia da Universidade Estadual de Maringá (UEM) e do Programa de Pós-Graduação em Economia (PCE/UEM). E-mail: jlparre@uem.br 


\section{Introdução}

O sistema agroindustrial cafeeiro é foco de diversos estudos devido a sua importância na história econômica e social do Brasil desde o período colonial. Esta commodity teve importante participação no acúmulo de divisas que deu início ao processo de industrialização do país. Além disso, a produção de café destaca-se pelo grande volume de exportação mundial, na capacidade de geração de emprego e, consequentemente, contribui significativamente para a renda nacional (VALE et al., 2006; SOUZA; PEROBELLI, 2007; TEIXEIRA; BERTELLA, 2015). Atualmente, o país é o principal produtor e exportador mundial de café, além de ser o segundo maior consumidor. Segundo o Ministério da Agricultura, Pecuária e Abastecimento (Mapa, 2017), a cafeicultura é o principal gerador de postos de trabalho na agropecuária nacional.

Ainda conforme a mesma fonte, a extensão territorial brasileira e as condições favoráveis do solo e do clima possibilitam a produção de diversos tipos de café, o que proporciona o atendimento de diferentes demandas. No total, a produção cafeeira conta com cerca de 287 mil produtores, predominando pequenos e miniprodutores, em, aproximadamente, 1900 municípios, distribuídos em 15 estados: Acre, Bahia, Ceará, Espírito Santo, Goiás, Distrito Federal, Mato Grosso, Mato Grosso do Sul, Minas Gerais, Pará, Paraná, Pernambuco, Rio de Janeiro, Rondônia e São Paulo. Dentre os diversos tipos do produto, segundo o Conselho Nacional do Café (CNC, 2017), suas duas principais espécies são o arábica e o robusta (conilon). O primeiro predomina nas lavouras de Minas Gerais, São Paulo, Bahia, Rio de Janeiro e em parte do Espírito Santo, enquanto a segunda espécie predomina nas lavouras do Espírito Santo, Rondônia, em parte da Bahia e de Minas Gerais (MINISTÉRIO DA AGRICULTURA, PECUÁRIA E ABASTECIMENTO, 2017).

Em relação às regiões, destaca-se a região sudeste, pois os três maiores estados produtores são dessa região. Minas Gerais, o maior produtor, é responsável por cerca de $50 \%$ da produção nacional, seguida por Espírito Santo e por São Paulo.

De acordo com a Companhia Nacional de Abastecimento (Conab, 2016), o estado de Minas Gerais continua despontando em primeiro lugar na produção brasileira anual de café, com 28,9 milhões de sacas em 2016. As duas principais regiões produtoras do estado são o sul de Minas Gerais (além do centro-oeste) e o cerrado mineiro (Triângulo, Alto Paranaíba e noroeste), que tiveram aumento da área e da produtividade e também da regularidade climática.

O Espírito Santo é o segundo maior produtor, com estimativa de produção de 9,1 milhões de sacas em 2016, o que representa uma queda de $14,5 \%$ na produção em relação a 2015. Conforme a Conab (2016), as lavouras de conilon foram as mais afetadas por questões climáticas, como a seca, as altas temperaturas, a má- 
distribuição de chuva e a insolação, o que implica redução de $30,7 \%$ em relação à safra passada. No entanto as lavouras de arábica tiveram crescimento de $28,2 \%$ devido à florada adequada e, assim, se recuperaram da baixa produção nos últimos dois anos (2015 e 2016).

O estado de São Paulo é o terceiro maior produtor, outrora líder da produção nacional, que deve produzir 5,9 milhões de sacas de café arábica em 2016. Esse resultado, registrado pela Conab (2016), deve-se principalmente às condições climáticas favoráveis no estado, pois as chuvas que ocorreram a partir de setembro de 2015 prolongaram-se satisfatoriamente até março de 2016 e proporcionaram uma excelente florada. Com isso, São Paulo teve área, produtividade e produção superior à safra passada, além da entrada de pés novos em produção, da melhoria nos tratos culturais e do retorno vigoroso das plantas manejadas na safra passada.

O Rio de Janeiro, quarto maior produtor, é formado por um parque cafeeiro com 41,76 milhões de plantas, 58,6 \% superior às existentes em 2015, das quais 2,64 milhões estão em formação e 39,12 milhões em produção de 6,4 mil covas. A área em formação, safra de 2016, aumentou para 820 hectares, superior em 817 hectares comparativamente à safra anterior, que foi de 3 hectares. O aumento expressivo das áreas em formação foi motivado pelos incentivos fornecidos pelo programa Rio Rural (CONAB, 2016).

O desenvolvimento das culturas agrícolas depende de diversas variáveis espaciais, como técnicas de produção, de clima, de solo e de topografia. Os efeitos de interdependência entre diferentes regiões podem ser observados de diversas formas, como ordenação geográfica dos dados, difusão espacial de certos fenômenos que influenciam os vizinhos, processos de competição espacial no âmbito de expansão da fronteira agrícola ou na formação de cinturões agrícolas (ALMEIDA; PEROBELLI; FERREIRA, 2008). Ainda nessa perspectiva, Teixeira e Bertella (2015) ressaltam que a agricultura é a atividade econômica mais sensível a esses efeitos espaciais.

Dessa forma, a análise de cluster ${ }^{4}$ e da convergência da produtividade média é de suma importância para analisar se determinada região de menor produtividade em certa cultura está crescendo a uma taxa mais elevada do que as regiões de maiores produtividades, servindo de base para formulação de políticas públicas locais voltadas para o desenvolvimento do setor agrícola.

Assim, o objetivo do trabalho é analisar a configuração espacial da produtividade média do café na região Sudeste do Brasil. Especificamente, objetiva-se identificar a existência de clusters nas microrregiões da região e verificar se houve

4 De acordo com Almeida et al. (2008), um cluster pode ser descrito como aglomeração espacial de atividades econômicas correlatas e que podem apresentar ganhos por estarem concentradas em uma região. Uma das maneiras de avaliar tal desempenho é acompanhar o comportamento da produtividade agrícola ao longo do tempo e das regiões. 
convergência de produtividade do café em nível microrregional nos anos de 1990, 1995, 2000, 2005, 2010 e 2015.

Este trabalho busca contribuir com a literatura recente, pois há poucos estudos que analisam a convergência da produtividade do café no Brasil utilizando a análise espacial. Na última década, não há análises acerca dessa temática. Desse modo, assolase a importância do aprofundamento do tema e da análise dado o aumento da produtividade do setor cafeeiro e da sua importância para a economia brasileira, em especial, na região Sudeste, maior produtora nacional.

$\mathrm{O}$ artigo divide-se em cinco seções, incluindo esta introdução. A seção seguinte apresenta o referencial empírico; a terceira explora detalhadamente a metodologia e o banco de dados utilizado; na quarta, são expostos os resultados da pesquisa; e, na última, são feitas as considerações finais.

\section{Referencial empírico}

Há uma gama de estudos que analisam a convergência de produtividade agrícola no Brasil e em outros países. O Quadro 1 apresenta os principais trabalhos desenvolvidos nos últimos anos.

Quadro 1 - Sinóptico da análise da convergência da produtividade agrícola.

\begin{tabular}{|c|c|c|}
\hline AUTOR & PROPÓSITO & RESULTADOS \\
\hline Lopes (2004) & $\begin{array}{l}\text { Analisar o comportamento da produtividade } \\
\text { da terra na agricultura brasileira, avaliando se } \\
\text { há ou não convergência na evolução dessa } \\
\text { variável. Período de } 1960 \text { a } 2001 \text {. Quatro } \\
\text { indicadores de convergência foram testados } \\
\text { que são: convergência- } \beta \text { absoluta, } \\
\text { convergência } \sigma \text {, grupos de convergência e } \\
\text { convergência- } \beta \text { condicional. }\end{array}$ & $\begin{array}{l}\text { Bons resultados econométricos foram obtidos } \\
\text { para a convergência- } \beta \text { condicional da } \\
\text { produtividade do algodão-herbáceo, da batata- } \\
\text { inglesa e do feijão. No entanto não se obteve } \\
\text { resultados satisfatórios para as culturas do arroz } \\
\text { e do milho. }\end{array}$ \\
\hline $\begin{array}{c}\text { Souza e } \\
\text { Perobelli (2007) }\end{array}$ & $\begin{array}{l}\text { Analisar a distribuição espacial do café nas } \\
\text { microrregiões brasileiras no período de } 1991 \text { a } \\
2003 \text {. }\end{array}$ & $\begin{array}{c}\text { Verificou a concentração espacial da produção } \\
\text { cafeeira nos estados de Minas Gerais e Espírito } \\
\text { Santo. }\end{array}$ \\
\hline $\begin{array}{l}\text { Oliveira Júnior, } \\
\text { Castelar e } \\
\text { Ferreira (2007) }\end{array}$ & $\begin{array}{l}\text { Analisar o processo de convergência } \\
\text { microrregional agrícola utilizando o Modelo } \\
\text { Threshold no período de } 1970 \text { a } 1996 .\end{array}$ & $\begin{array}{l}\text { Os resultados mostraram a existência de três } \\
\text { clubes de convergência. Um formado pelo } \\
\text { grupo de microrregiões mais ricas, outro pelo } \\
\text { grupo de microrregiões mais pobres e um } \\
\text { terceiro grupo intermediário. Os resultados } \\
\text { também mostraram que o capital físico é mais } \\
\text { importante que o capital humano na explicação } \\
\text { do processo de crescimento da agricultura } \\
\text { brasileira. }\end{array}$ \\
\hline & & Continua \\
\hline
\end{tabular}




\begin{tabular}{|c|c|c|}
\hline \multicolumn{3}{|l|}{ Continuação } \\
\hline $\begin{array}{c}\text { Almeida, } \\
\text { Perobelli e } \\
\text { Ferreira (2008). }\end{array}$ & $\begin{array}{c}\text { Analisar a convergência espacial da } \\
\text { produtividade agrícola da terra para as } \\
\text { microrregiões brasileiras entre o período de } \\
1991 \text { a } 2003 \text {. }\end{array}$ & $\begin{array}{l}\text { Preliminarmente, em uma análise exploratória } \\
\text { de dados espaciais, detectou-se a presença de } \\
\text { autocorrelação espacial para a produtividade da } \\
\text { terra. }\end{array}$ \\
\hline $\begin{array}{l}\text { Fochezatto e } \\
\text { Stülp (2008) }\end{array}$ & $\begin{array}{l}\text { Analisar a convergência da produtividade do } \\
\text { trabalho da agropecuária brasileira na década } \\
\text { de noventa, comparando-a com outros setores } \\
\text { e projetando seu comportamento relativo } \\
\text { usando cadeias de Markov. }\end{array}$ & $\begin{array}{l}\text { Os resultados indicaram que a produtividade do } \\
\text { trabalho na agropecuária não está convergindo, } \\
\text { sendo que alguns estados evoluem para o nível } \\
\text { superior de produtividade e outros para o nível } \\
\text { inferior. Em geral, o tempo de convergência foi } \\
\text { bastante longo, indicando que os fatos } \\
\text { econômicos do período em questão não tiveram } \\
\text { maiores repercussões sobre esse processo. }\end{array}$ \\
\hline $\begin{array}{l}\text { Diniz; Câmara; } \\
\text { Massambani; } \\
\text { Anhesinie } \\
\text { Sesso Filho } \\
\text { (2012) }\end{array}$ & $\begin{array}{l}\text { Analisar a evolução da produtividade média do } \\
\text { setor citrícola no estado de São Paulo e } \\
\text { verificar ganhos de produtividade. A } \\
\text { metodologia utilizada é a análise exploratória } \\
\text { de dados espaciais no período de } 2002 \text { a } 2010 \text {. }\end{array}$ & $\begin{array}{l}\text { O estudo concluiu que, em geral, os municípios } \\
\text { com alta produtividade média na produção de } \\
\text { laranja são vizinhos de outros municípios que } \\
\text { apresentam o mesmo padrão produtivo. }\end{array}$ \\
\hline $\begin{array}{c}\text { Teixeira; } \\
\text { Bertella (2015) }\end{array}$ & $\begin{array}{c}\text { Analisar a distribuição espacial da } \\
\text { produtividade média do café entre as } \\
\text { microrregiões mineiras durante o período de } \\
\text { 1997-2006. }\end{array}$ & $\begin{array}{l}\text { O estudo concluiu que a produtividade média } \\
\text { do café não segue um processo aleatório, } \\
\text { ocorrendo um reordenamento espacial. Além } \\
\text { disso, verificou-se que a produtividade está se } \\
\text { distribuindo de forma mais homogênea em } \\
\text { Minas Gerais. }\end{array}$ \\
\hline $\begin{array}{c}\text { Favro; } \\
\text { Marconato; } \\
\text { Alves (2016) }\end{array}$ & $\begin{array}{l}\text { Analisar a distribuição geográfica e a } \\
\text { produtividade do milho para os anos de } 1994 \text {, } \\
\text { 2000, 2004, } 2010 \text { e } 2014 \text {, nos principais estados } \\
\text { produtores brasileiros. }\end{array}$ & $\begin{array}{l}\text { O estudo apontou a existência de } \\
\text { autocorrelação espacial positiva durante todo o } \\
\text { período analisado, assim como a constatação de } \\
\text { que houve convergência produtiva em 2004- } \\
\text { 2000; 2010-2004 e 2014-2010. }\end{array}$ \\
\hline
\end{tabular}

Fonte: Elaboração própria.

Os trabalhos citados mostram a importância de estudos relacionados à análise espacial e a convergência, especificamente, da produtividade na agricultura. Observa-se que apenas dois trabalhos analisaram a cultura do café. Enquanto Souza e Perobelli (2007) analisam as microrregiões brasileiras, Teixeira e Bertella (2015) verificam a distribuição espacial apenas das microrregiões mineiras. Ambos os estudos verificaram a formação de clusters, o primeiro observa uma forte concentração da produtividade do setor cafeeiro nos estados de Minas Gerais e no Espírito Santo. Em contrapartida, Teixeira e Bertella (2015) verificaram que está ocorrendo um reordenamento espacial das concentrações da produção de café nas microrregiões mineiras. Outro ponto a se destacar nos trabalhos analisados é que a 
maioria utiliza indicadores de produtividade parcial, seja da terra ou do trabalho, em virtude da dificuldade de obter valores de capital nas estatísticas sobre a agricultura brasileira. Nesse sentido, na mesma linha dos artigos revisados, o presente estudo também utiliza um indicador de produtividade da terra do setor cafeeiro. Para o cálculo da produtividade total dos fatores, são necessárias informações sobre todos os insumos utilizados no processo de produção, os quais não estão disponíveis em nível de microrregiões para os períodos analisados.

\section{Metodologia e dados}

Para atingir os objetivos propostos, utilizam-se as técnicas de econometria espacial. Conforme Pinheiro, Neto e Parré (2007), os estudos que usam a econometria espacial consideram dois efeitos espaciais na sua estimação: o primeiro é a dependência espacial ou autocorrelação espacial e, o segundo, a heterogeneidade espacial. Para compreender melhor esses efeitos, são abordados a Análise Exploratória de Dados Espaciais (Aede), por meio da utilização do Índice de Moran Local e Global, utilizados para identificar a autocorrelação e a econometria espaciais através do modelo de convergência espacial.

\subsection{Análise exploratória de dados espaciais (Aede)}

A Aede está baseada nos efeitos decorrentes da dependência e da heterogeneidade espacial. Através desse método, é possível descrever a distribuição espacial de determinada variável, os padrões de associações espaciais (clusters espaciais), a identificação de localidades atípicas (outliers) e a verificação da existência de diferentes regimes espaciais ou outras formas de instabilidade espacial (ANSELIN, 1988).

Segundo Almeida (2012), a dependência espacial é dada pela interação dos agentes no espaço, ou seja, o valor de uma variável de interesse numa certa região $i$ tende a estar correlacionada ao valor dessa variável em análise nas regiões vizinhas $j$. O autor também ressalta que esses dois efeitos, dependência e heterogeneidade espacial, estão extremamente associados, pois a heterogeneidade espacial gera dependência e, por sua vez, esta pode levar à heterogeneidade.

No entanto, para implementar a Aede e aplicar as técnicas de econometria espacial, Anselin (1988) frisa que a variável analisada deve ser intensiva ou espacialmente densa. Além disso, deve-se definir uma matriz de peso espacial (W) a ser utilizada como forma de expressar o arranjo espacial que represente a proximidade geográfica entre as regiões estudadas. 
Posteriormente, a identificação da presença de autocorrelação espacial é fornecida pelas medidas globais e locais, tais como as estatísticas I de Moran Global e I de Moran Local.

\subsection{Determinação da matriz de pesos espaciais (W)}

A matriz de pesos é a forma de expressar a estrutura espacial dos dados, ou seja, descreve a estrutura de dependência entre as regiões de análise e é o ponto inicial para qualquer teste estatístico ou modelo espacial (PEROBELLI, et al., 2008).

$\mathrm{Na}$ literatura, há diversas matrizes de pesos espaciais. O grau de conexão expresso nas matrizes de pesos espaciais pode ser classificado por critérios geográficos ou socioeconômicos (ALMEIDA, 2012).

Dentre as matrizes de pesos com critérios geográficos, as matrizes mais comuns são a convenção rainha (queen) e torre (rock). Enquanto a primeira considera como vizinhas as localidades que possuem fronteira comum, além de nós (vértices); a segunda, considera como vizinhas somente as localidades que possuem fronteira comum (TEIXEIRA; BERTELLA, 2015).

Outra maneira de se expressar a estrutura de conectividade é por meio da distância geográfica, especificamente pela matriz de peso denominada $k$ vizinhos que se baseia na ideia dos $k$ vizinhos mais próximos (ALMEIDA, 2012).

Almeida et al. (2008) ressalta que a escolha da matriz de pesos espaciais é de extrema importância em uma Aede dado que os resultados da análise são sensíveis a tal seleção. Embora não haja um critério definitivo de qual matriz de peso é a mais adequada para utilizar em cada problema, Baumont (2004 apud ALMEIDA, 2012) sugere que se escolha a matriz que apresente o maior valor do teste $I$ de Moran e que seja significativa estatisticamente. 


\subsection{Autocorrelação espacial global}

Segundo Almeida (2012), o primeiro coeficiente de autocorrelação espacial foi proposto por Moran em 1948 e ficou conhecido como a estatística I de Moran, a qual utiliza a medida de autocovariância na forma de produto cruzado.

A fórmula estatística do $I$ de Moran é representada pela equação:

$$
I=\frac{n}{\sum \sum w_{i j}} \frac{\sum \sum w_{i j} z_{i} z_{j}}{\sum\left(z_{i}\right)^{2}}(1),
$$

em que $n$ é o número de unidades espaciais; zé a variável de interesse e $w_{i j}$ é o peso espacial para o par de unidades espaciais $i$ e $j$, medindo o grau de interação entre elas.

Os valores desse coeficiente variam entre um e menos um, em que "menos um" representa correlação linear perfeitamente negativa e "um" indica uma correlação linear perfeitamente positiva (TEIXEIRA; BERTELLA, 2015).

Além disso, o nível de significância do $I$ de Moran reforça a informação sobre os dados estarem distribuídos aleatoriamente e indica se os dados estão concentrados através das regiões (ALMEIDA, 2012).

\subsection{Autocorrelação espacial local}

Para verificar a existência de clusters espaciais locais de valores altos e baixos e quais regiões mais contribuem para a existência de autocorrelação espacial, deve-se implementar medidas de autocorrelação espaciais locais. Dentre elas, está o diagrama de dispersão de Moran e os indicadores locais de associação espacial (Lisa) (TEIXEIRA; BERTELLA, 2015).

A Lisa indica o grau de autocorrelação espacial local, algebricamente expressa por:

$$
I_{i}=Z_{i} \sum_{j=1}^{j} W_{i j} Z_{j}(2)
$$

Segundo Almeida et al. (2008), esses indicadores devem satisfazer dois critérios: possuir, para cada observação, uma indicação de clusters espaciais significativos de valores similares ao redor de cada observação, e, além disso, o somatório dos indicadores Lisa devem ser proporcionais ao indicador de autocorrelação espacial global. 


\subsection{Diagrama de dispersão de Moran}

O diagrama de dispersão de Moran é uma ferramenta gráfica para analisar o $I$ de Moran. O valor da variável em análise está colocado no eixo das abscissas e a defasagem espacial da variável de interesse está no eixo das ordenadas. O diagrama é composto por quatro quadrantes: Alto-Alto (AA); Baixo-Baixo (BB); Alto-Baixo $(\mathrm{AB})$ e Baixo-Alto (BA) que correspondem aos padrões de associação local espacial entre as regiões e os seus vizinhos (ALMEIDA et al., 2008).

Figura 1 - Diagrama de dispersão de Moran

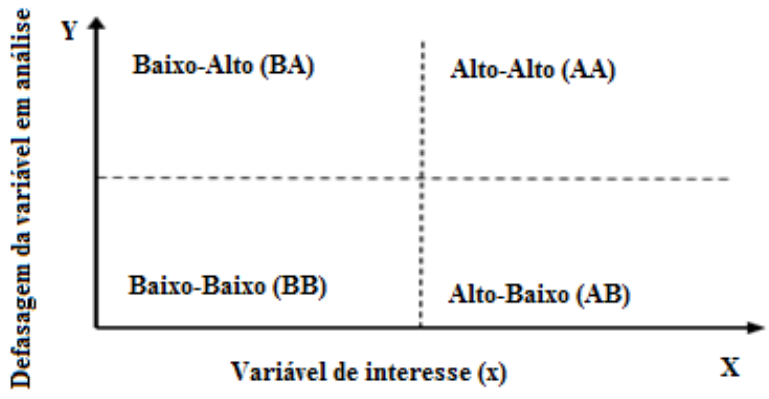

Fonte: Elaboração própria com base em Almeida (2012).

$\mathrm{O}$ primeiro e o terceiro quadrante, $\mathrm{AA}$ e $\mathrm{BB}$, respectivamente, apresentam autocorrelação espacial positiva, ou seja, essas regiões formam clusters de valores similares; AA composto por valores altos e BB valores baixos. Por outro lado, o segundo e o quarto quadrante, $\mathrm{BA}$ e $\mathrm{AB}$, apresentam autocorrelação espacial negativa, isto é, formam clusters com valores distintos (ALMEIDA et al., 2008).

\subsection{Métodos econométricos}

Ao tratar de problemas que envolvam efeitos de transbordamento entre as regiões, deve-se considerar explicitamente componentes espaciais em sua forma funcional (ALMEIDA et al., 2008). No presente artigo, estimam-se: o modelo clássico de regressão linear pelos métodos Mínimos Quadrados Ordinários (MQO), Defasagem Especial (SAR) e Erro Espacial (SEM).

Para definir a melhor especificação do modelo de convergência, considerando a presença de autocorrelação espacial, segue-se as recomendações de Florax, Folmer e Rey (2003) e Anselin (2005) apresentadas em Teixeira e Bertella (2015):

a) estimar o modelo clássico de regressão linear por MQO; 
b) testar a hipótese de ausência de autocorrelação espacial devido a uma defasagem ou a um erro por meio do valor do multiplicador de Lagrange para defasagem espacial (ML $\rho$ ) e Multiplicador de Lagrange para o erro espacial $\left(M L_{\lambda}\right)$;

c) se ambos os testes não sejam significativos, a utilização do modelo clássico é mais adequada. Caso contrário, recomenda-se utilizar o passo d;

d) caso ambos sejam significativos, estima-se o modelo apontado como o mais significativo de acordo com as versões robustas desses testes, ou seja, o multiplicador de Lagrange robusto para a defasagem espacial (MLR $\rho$ ) e o multiplicador de Lagrange robusto para o erro espacial $M L R_{\lambda}$. Caso MLR $\rho>M L R \lambda$, o modelo mais adequado será o modelo com defasagem espacial. Caso contrário, o mais apropriado a ser adotado é o modelo de erro espacial.

Ainda segundo os autores, a estimação do modelo de $\beta$ convergência por MQO busca apenas identificar a melhor maneira de estimar a seguinte equação:

$$
\ln \left(\frac{P m e_{t}}{P m e_{t-n}}\right)=\alpha+\beta \ln \left(P m e_{t-n}\right)+u_{i}(3)
$$

Onde $\ln \left(\frac{P m e_{t}}{P m e_{t-n}}\right)$ é o logaritmo natural da razão entre a produtividade média entre dois anos em análise, $\ln \left(P m e_{t-n}\right)$ é o logaritmo natural da variável em análise no período inicial e $u_{i}$ é o termo de erro.

De acordo com Lopes (2004), a convergência é um processo em que uma mesma variável apresenta diferentes valores entre unidades espaciais diferentes, mas essa diferença se reduz ao longo do tempo, indicando que a desigualdade ou discrepância entre os valores da variável analisada diminui ou é eliminada.

- Modelo de defasagem espacial (spatial lag model - SAR)

O SAR sugere que a autocorrelação espacial é decorrente da interação entre as unidades espaciais em análise. Segundo Almeida (2012), a defasagem espacial é considerada como variável independente na equação de convergência. Dessa forma, o SAR é representado pela seguinte equação:

$$
\ln \left(P e_{t} / P m e_{t-n}\right)=\alpha+\beta \ln \left(P m e_{t-n}\right)+\rho W \ln \left(P m e_{t} / P m e_{t-n}\right)+\varepsilon_{t}(4)
$$


em que $\rho$ representa o coeficiente de defasagem espacial. Espera-se que $\rho>0$, sugerindo a existência de autocorrelação espacial positiva. $O$ elemento $\rho W \ln \left(\right.$ Pme $_{t} /$ Pme $\left._{t-n}\right)$ pode ser entendido como a média dos valores da taxa de crescimento das regiões vizinhas.

- Modelo de erro espacial (spatial error model - SEM)

Segundo Almeida (2012), no SEM, ocorre a introdução de um termo de erro autorregressivo, definido por

$$
u_{i}=(I+\lambda W)+\varepsilon(5)
$$

onde $\lambda$ representa o coeficiente escalar do parâmetro do erro autorregressivo espacial e Wrepresenta a defasagem espacial. Dessa forma, o modelo de erro espacial é representado pela equação

$$
\ln \left(P m e_{t} / P m e_{t-n}\right)=\alpha+\beta \ln \left(P m e_{t-n}\right)+(I+\lambda W)+\varepsilon(6)
$$

O modelo SEM por especificar a dependência espacial, apenas no termo de erro evita problemas de consistência, ocorrendo apenas o risco de problemas de eficiência (ALMEIDA, 2012).

\subsection{Base de dados}

Os dados utilizados foram obtidos da Pesquisa Agrícola Municipal do Instituto Brasileiro de Geografia e Estatística (IBGE), consultados por meio do Sistema IBGE de Recuperação Automática (Sidra). A região analisada é o Sudeste, formada pelos estados de Minas Gerais, São Paulo, Rio de Janeiro e Espírito Santo que totalizam 160 microrregiões. Para o desenvolvimento da análise foram analisados dados sobre a produtividade do café referente à quantidade produzida (em quilogramas) por área colhida (em hectares) para os anos de 1990, 1995, 2000, 2005, 2010 e 2015. Ou seja, a variável "produtividade" refere-se à produtividade parcial da terra.

\section{Análise dos resultados}

Geograficamente, a produtividade do café, em todos os anos analisados, está distribuída conforme visualização nos mapas de distribuição espacial representados na Figura 2. As regiões em tons azuis representam as microrregiões em que há produtividade abaixo da média. Já as microrregiões em tons vermelhos tiveram 
produtividade acima da média. A produtividade média do café nas microrregiões do Sudeste brasileiro teve sua configuração alterada durante o período analisado, principalmente nos estados de São Paulo e de Minas Gerais. Essa modificação na configuração da produtividade mineira também foi verificada por Teixeira e Bertella (2015) no período de 1997-2007.

A pior produtividade média foi observada no ano de 2005 , cuja representação foi de apenas $887 \mathrm{~kg} / \mathrm{ha}$, sendo que em 18 microrregiões não houve produção. Nos outros dois anos analisados, 2010 e 2015, ocorreu uma melhora na produtividade média passando para $1109 \mathrm{~kg} / \mathrm{ha}$ e $1141 \mathrm{~kg} / \mathrm{ha}$, respectivamente.

Figura 2 - Mapas da distribuição espacial (em desvios-padrão) da produtividade média do café para as microrregiões do Sudeste brasileiro nos anos de 1990, 1995, 2000, 2005,2010 e 2015

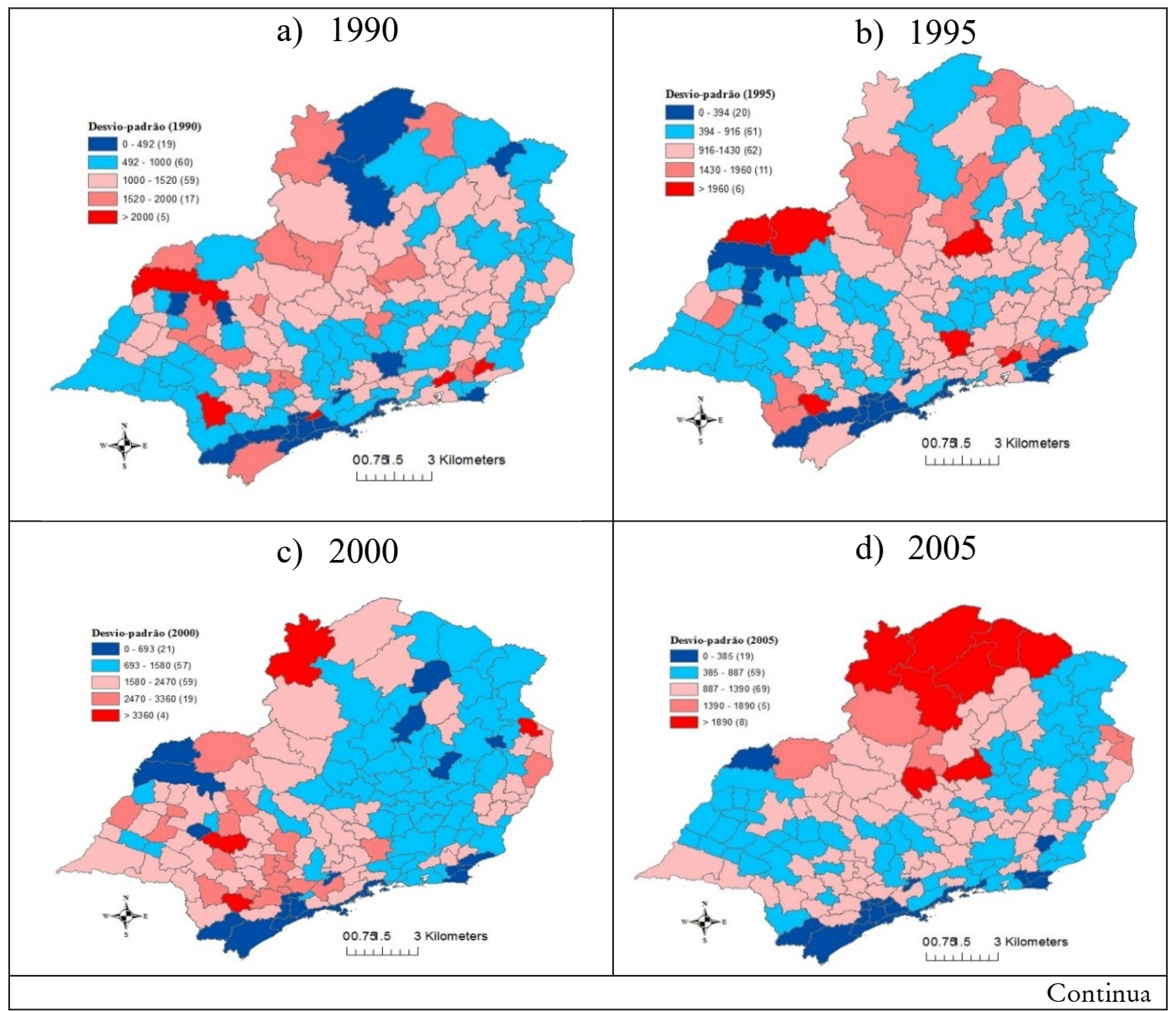




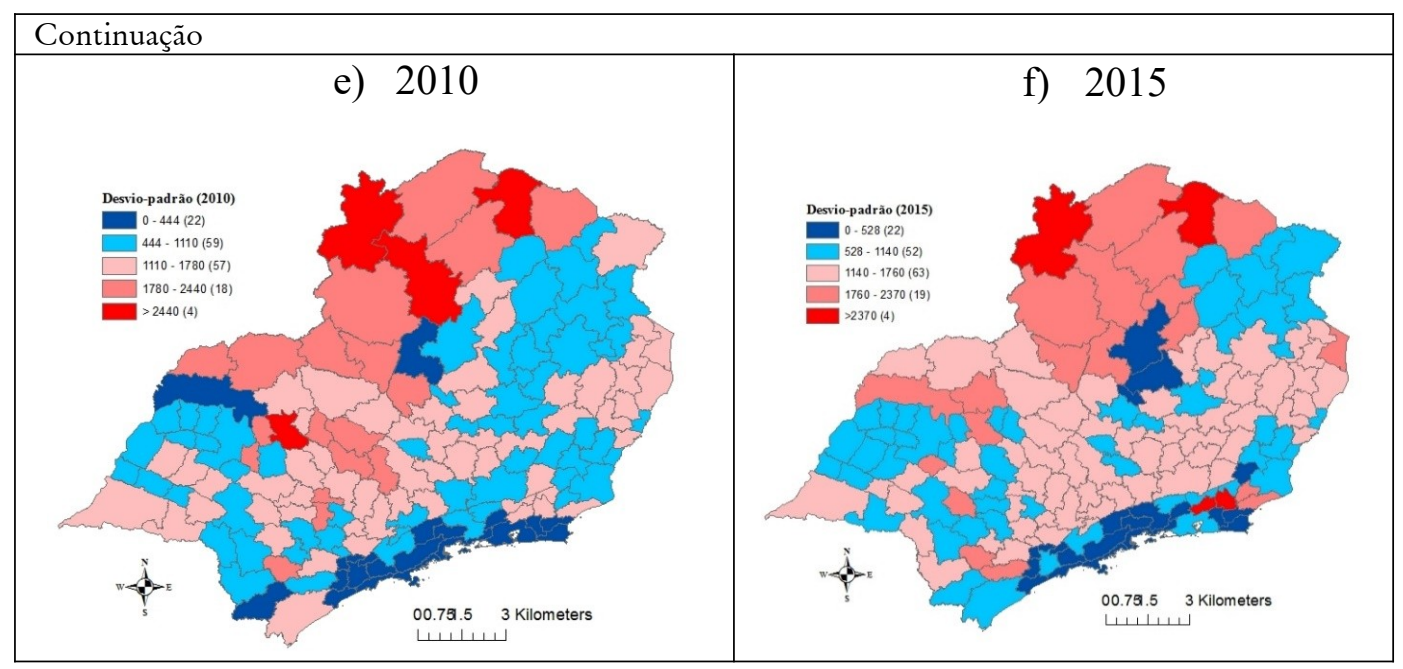

Fonte: Elaboração própria com base no software GeoDa.

Para uma análise mais completa sobre a convergência da produtividade, assim como a influência de uma localidade sobre sua vizinhança e vice-versa, é necessário verificar a aleatoriedade espacial e aplicar o modelo de convergência (TEIXEIRA; BERTELLA, 2015).

O primeiro passo na análise de Aede é a estimação da estatística $I$ de Moran para a produtividade das microrregiões, a qual revela a presença de autocorrelação espacial entre as distintas microrregiões. A Tabela 1 refere-se ao $I$ de Moran ${ }^{5}$ global para o período da análise.

Tabela 1 - I de Moran: teste de autocorrelação espacial

\begin{tabular}{cccc}
\hline Ano & I de Moran & Média & p-valor \\
\hline 1990 & 0,1706 & $-0,0060$ & 0,0020 \\
1995 & 0,2864 & $-0,0048$ & 0,0010 \\
2000 & 0,2897 & $-0,0055$ & 0,0010 \\
2005 & 0,4964 & $-0,0053$ & 0,0010 \\
2010 & 0,4372 & $-0,0066$ & 0,0010 \\
2015 & 0,3576 & $-0,0073$ & 0,0010 \\
\hline
\end{tabular}

Fonte: Elaboração própria.

Nota: Todas as estimações são baseadas em 999 permutações aleatórias.

Em todos os anos analisados, os valores foram positivos e significativos, indicando que existe autocorrelação espacial global, ou seja, as microrregiões de alta

5 Foram realizados os testes para as convenções de matrizes espaciais: torre, k2, k5, k10 e k15, porém a convenção rainha apresentou os melhores resultados. 
produtividade média de café estão cercadas por regiões com as mesmas características. $\mathrm{O}$ aumento gradual dessa estatística indica que a dependência espacial entre as microrregiões analisadas aumentou nesse período.

Dado que a estatística $I$ de Moran apenas nos indica se há autocorrelação espacial, é necessário o uso da estatística Lisa que não fornece a configuração espacial da produtividade média do café. A Figura 3 apresenta os clusters da produtividade média do café durante os quinquênios de 1990 e 2015.

Figura 3 - Clusters da produtividade média do café

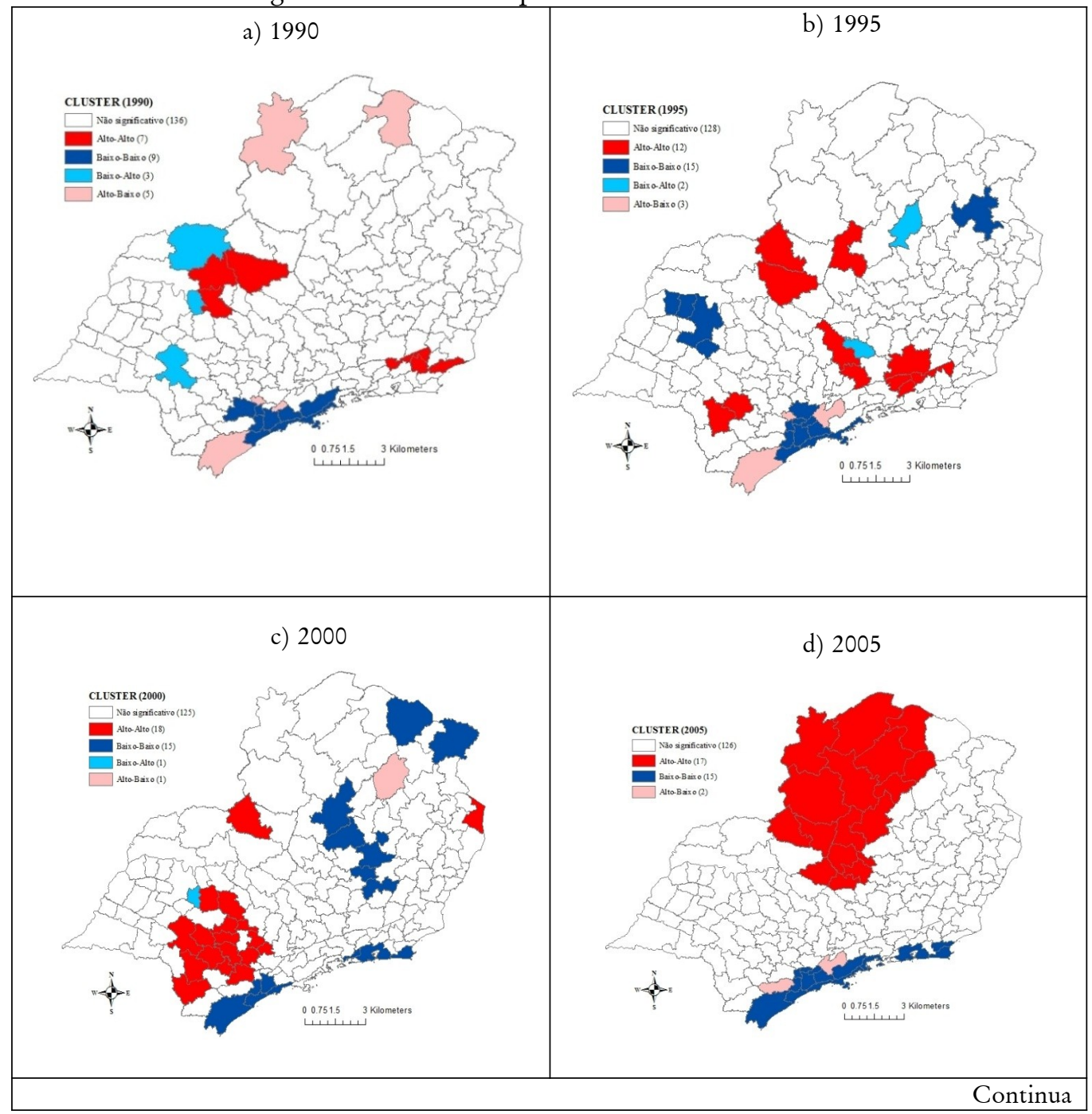




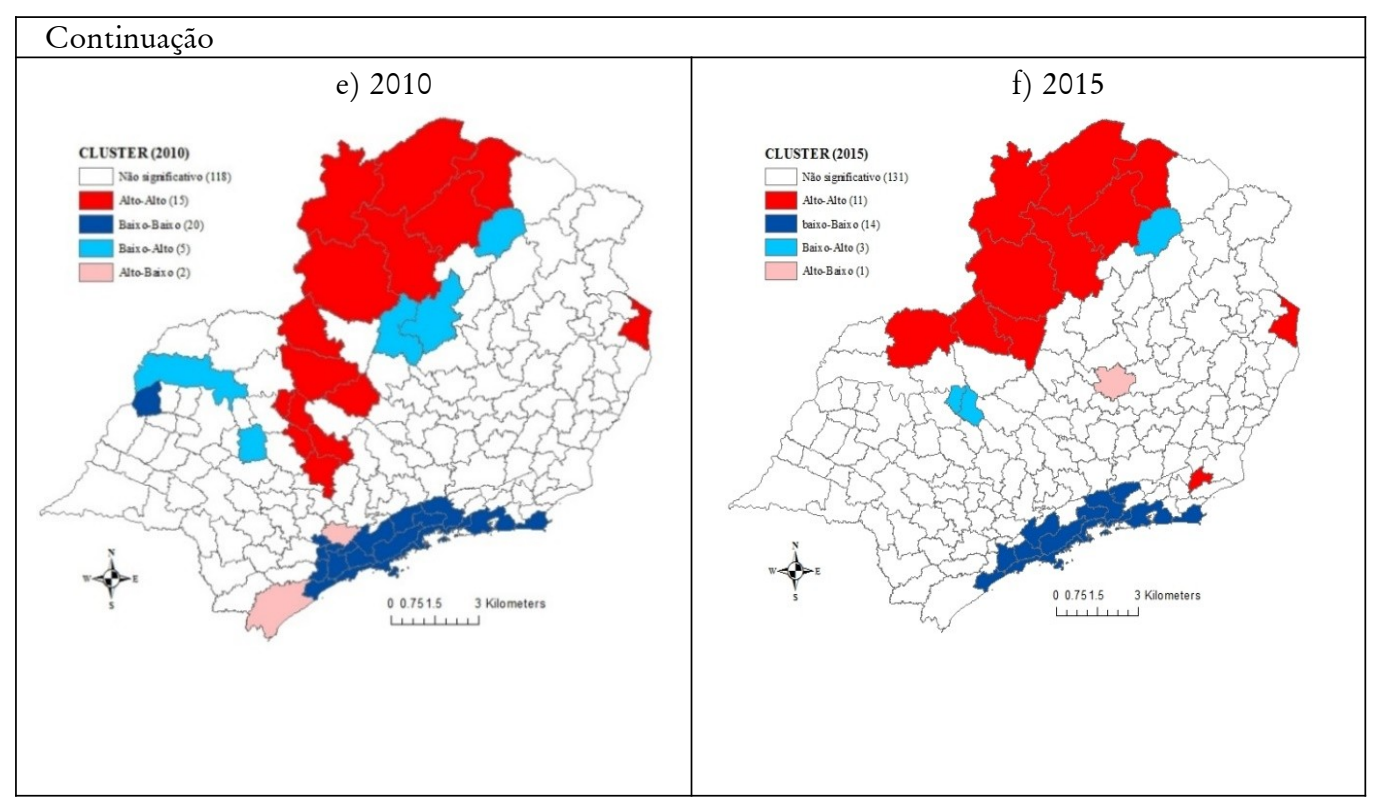

Fonte: Elaboração própria.

As aglomerações destacadas em vermelho são os clusters Alto-Alto e em azul estão os conjuntos Baixo-Baixo. Com exceção do ano de 2005, todos os anos analisados apresentam os quatro tipos de associações espaciais possíveis. De 1990 a 2000, pôde-se verificar que não existe um padrão na distribuição da produtividade do café na região Sudeste e que, de 2005 a 2015, nos agrupamentos de AA e BB não houve mudanças muito significativas.

Em 1990, as microrregiões de Piedade, Sorocaba, Paraitinga, Caraguatatuba, Osasco, Itapecerica da Serra, Santos e o litoral sul paulista representavam os clusters BB, enquanto Uberaba (MG), Araxá (MG), São Joaquim da Barra (SP), Macaé (RJ), Três Rios (RJ), Cantagalo-Cordeiro (RJ), Nova Friburgo (RJ) configuravam os clusters AA.

No ano de 1995, o cluster Alto-Alto era representado pelas microrregiões de Patrocínio (MG), Araxá (MG), Pará de Minas (MG), Varginha (MG), São Lourenço (MG), Juiz de Fora (MG), Três Rios (RJ), Barra do Piraí (RJ), Vassouras (RJ), Avaré (SP), Botucatu (SP), embora Três Marias (MG) não possa se configurar como parte do cluster por estar isolada no mapa.

Já em 2005, as microrregiões de Minas Gerais que compunham o cluster AA era Uná, Paracatu, Januária, Janaúba Pirapora, Montes Claros, Grão Mogol, Bocaiúva, Patrocínio, Patos de Minas, Três Marias, Curvelo, Pará de Minas, Piuí, Formiga, Divinópolis, Bom Despacho. Por outro lado, o cluster BB, de baixa produtividade, é representado pelas microrregiões de Lagos (RJ), Rio de Janeiro (RJ), Paraibuna (SP), Caraguatatuba (SP), Registro (SP), Itanhaém (SP), Osasco (SP), Franco da Rocha 
(SP), Guarulhos (SP), Itapecerica da Serra (SP), São Paulo (SP), Mogi das Cruzes (SP), Santos (SP) e Bacia de São João (SP).

O mapa referente ao ano de 2010 mostra que as microrregiões de Unaí (MG), Paracatu (MG), Januária (MG), Janaúba (MG), Pirapora (MG), Montes Claros (MG), Patrocínio (MG), Araxá (MG), Piuí (MG), Formiga (MG), São Sebastião do Paraíso (MG), Ituverava (SP), Franca (SP), Batatais (SP) e São João da Boa Vista (SP) formam o cluster AA, enquanto as microrregiões de Lagos (RJ), Vale do Paraíba Fluminense (RJ), Baía da Ilha Grande (RJ), Macacu-Caceribu (RJ), Rio de Janeiro (RJ), Jales (SP), Jundiaí (SP), São José dos Campos (SP), Guaratinguetá (SP), Bananal (SP), Paraibuna (SP), Itanhaém (SP), Osasco (SP), Franco da Rocha (SP), Itapecerica da Serra (SP), São Paulo (SP), Mogi das Cruzes (SP) e Santos (SP), o cluster BB.

Por último, no mapa referente ao ano de 2015, o cluster AA foi formado pelas microrregiões de Unaí (MG), Paracatu (MG), Januária (MG), Janaúba (MG), Pirapora (MG), Montes Claros (MG), Uberlândia (MG), Patrocínio (MG), Patos de Minas (MG), Macaé (RJ), Santa Maria Madalena (RJ) enquanto as microrregiões paulistas de São José dos Campos, Bananal, Paraibuna, Caraguatatuba, Itanhaém, Osasco, São Paulo, Mogi das Cruzes e Santos compõem a formação do cluster BB.

$\mathrm{O}$ aumento da produtividade média ${ }^{6}$ de café, nas microrregiões do norte e do noroeste de Minas Gerais, entre 2005 e 2015, em grande parte, é consequência dos programas de desenvolvimento agrícola implementados no Triângulo Mineiro/Alto Paranaíba. Esses programas tornaram a agricultura mais moderna, com altos índices de produtividade na cafeicultura como nas demais culturas frutos de uma política agrícola "modernizadora e conservadora", a qual buscava a expansão e o aumento contínuo da área cultivada e da produtividade pela inserção de métodos modernos e de alta tecnologia no campo (DO VALE; CALDERARO; FAGUNDES, 2014).

Ademais, segundo Veríssimo (2015, p. 93), essa expansão foi possível por causa das características favoráveis do solo, do clima e dos altos investimentos internos e externos, "favorecendo a infraestrutura para a instalação de grandes empresas nacionais e multinacionais ligados ao setor agropecuário.”

Por outro lado, a configuração do cluster BB observada desde 2000, em grande parte, é explicada pelas transformações das estruturas produtivas, nas quais não há mais o predomínio da monocultura, ou seja, passa a predominar o cultivo de mais de um produto agrícola, dentre eles, o café.

Para a análise de convergência, adotou-se os procedimentos citados na seção 5.7. A Tabela 2 apresenta os resultados da estimação do modelo de convergência absoluta, representados pela Equação 3 .

6 Utiliza-se produtividade média, no presente estudo, como a média da quantidade produzida (em quilogramas) por área colhida (em hectares). 
Convém mencionar que esse método estuda a hipótese de que as microrregiões com baixa produtividade média do café tendem a elevar a produtividade média mais rapidamente que microrregiões com alta produtividade média (TEIXEIRA et al., 2015).

Os resultados indicam que houve convergência em todos os subperíodos analisados, dado que o valor do coeficiente $\beta$ foi negativo e significativo a $1 \% \mathrm{em}$ todas as estimações. $O$ diagnóstico do teste do Multiplicador de Lagrange de defasagem e do erro e do Multiplicador de Lagrange robusto permitem inferir qual o melhor modelo econométrico a ser utilizado, assim como a existência de autocorrelação espacial (ALMEIDA et al., 2008).

Tabela 2 - Resultados do Modelo de Mínimos Quadrados Ordinários

\begin{tabular}{|c|c|c|c|c|c|c|}
\hline \multicolumn{7}{|c|}{ Modelo Clássico - MQO } \\
\hline Período & $1990-1995$ & $1995-2000$ & $2000-2005$ & $2005-2010$ & $2010-2015$ & $1990-2015$ \\
\hline \multicolumn{7}{|l|}{ Coeficientes } \\
\hline \multirow[t]{2}{*}{ A } & $-0,0592$ & 0,4252 & $-0,1297$ & 0,6927 & 0,0988 & 0,1645 \\
\hline & $(0,0644)$ & $(0,0000)$ & $(0,0003)$ & $(0,0000)$ & $(0,0000)$ & $(0,0000)$ \\
\hline \multirow[t]{2}{*}{ B } & $-0,5437$ & $-0,7114$ & $-0,8129$ & 0,8435 & -0.4792 & $-0,7954$ \\
\hline & $(0,0000)$ & $(0,0000)$ & $(0,0000)$ & $(0,0000)$ & $(0,0000)$ & $(0,0000)$ \\
\hline \multicolumn{7}{|l|}{ Estatísticas } \\
\hline LIK & $-79,763$ & $-100,113$ & $-49,943$ & $-46,971$ & $-26,489$ & $-57,597$ \\
\hline AIC & 163,526 & 204,226 & 103,887 & 97,942 & 56,978 & 119,194 \\
\hline $\mathrm{SC}$ & 169,677 & 210,377 & 110,037 & 104,092 & 63,128 & 125,345 \\
\hline Condition Number & 1,090 & 1,155 & 2,226 & 1,196 & 1,444 & 1,090 \\
\hline \multirow[t]{2}{*}{ Teste JB } & 196,5562 & 3,192 & 42,764 & 34,597 & 1049,307 & 355,412 \\
\hline & $(0,0000)$ & $(0,2027)$ & $(0,0000)$ & $(0,0000)$ & $(0,0000)$ & $(0,0000)$ \\
\hline \multirow[t]{2}{*}{ Teste BP } & 32,575 & 0,943 & 0,205 & 6,027 & 1,938 & 12,731 \\
\hline & $(0,0382)$ & $(0,3314)$ & $(0,6508)$ & $(0,0141)$ & $(0,1639)$ & $(0,0004)$ \\
\hline \multirow[t]{2}{*}{$I$ de Moran } & 0,2957 & 0,4189 & 0,4125 & 0,4811 & $-0,0859$ & 0,1108 \\
\hline & $(0,0000)$ & $(0,0000)$ & $(0,0000)$ & $(0,0000)$ & $(0,0455)$ & $(0,0131)$ \\
\hline \multirow[t]{2}{*}{ ML defasagem } & 27,233 & 92,887 & 52,695 & 69,371 & 1,373 & 2,522 \\
\hline & $(0,0000)$ & $(0,0000)$ & $(0,0000)$ & $(0,0000)$ & $(0,2413)$ & $(0,1123)$ \\
\hline \multirow[t]{2}{*}{ MLR da defasagem } & 2,707 & 17,935 & 2,635 & 0,863 & 0,501 & 0,562 \\
\hline & $(0,0999)$ & $(0,0000)$ & $(0,1045)$ & $(0,3529)$ & $(0,4793)$ & $(0,4533)$ \\
\hline \multirow[t]{2}{*}{ ML do erro } & 37,3811 & 75,049 & 72,751 & 98,980 & 3,152 & 5,247 \\
\hline & $(0,0000)$ & $(0,0000)$ & $(0,0000)$ & $(0,0000)$ & $(0,0758)$ & $(0,0220)$ \\
\hline \multirow[t]{2}{*}{ MLR do erro } & 12,8554 & 0,097 & 22,691 & 30,471 & 2,280 & 3,287 \\
\hline & $(0,0003)$ & $(0,7555)$ & $(0,0000)$ & $(0,0000)$ & $(0,1311)$ & $(0,0698)$ \\
\hline
\end{tabular}

Fonte: Elaboração própria.

Nota: Os valores entre parênteses correspondem ao p-valor. 
Pela análise das estatísticas dos multiplicadores de Lagrange, apenas o multiplicador de Lagrange do erro robusto mostrou-se significativo para os subperíodos de 1990/1995, 2000/2005, 1990/2015. O multiplicador de Lagrange de defasagem foi significativa apenas no subperíodo de 1995/2000. Portanto o modelo de erro espacial é o mais indicado para explicar o processo de convergência absoluta da produtividade do café dado que o ML do erro é significativo a $1 \%$ nos subperíodos mencionados.

Contudo, para melhor compreensão dos resultados e comparação dos métodos, foram estimados os dois modelos propostos, SAR e SEM, para todos os subperíodos em análise. Entretanto é importante ressaltar que o resultado do teste de Jarque-Bera, apresentado na Tabela 2, indica que os erros não são distribuídos normalmente, portanto a estimação dos modelos de erro espacial e defasagem espacial serão pelo método de dois estágios para a correção desse problema.

Tabela 3 - Modelos econométricos espaciais de defasagem (SAR) e erro (SEM)

\begin{tabular}{|c|c|c|c|c|c|c|c|c|c|c|c|c|}
\hline & \multicolumn{2}{|c|}{ 1990-1995 } & \multicolumn{2}{|c|}{$1995-2000$} & \multicolumn{2}{|c|}{$2000-2005$} & \multicolumn{2}{|c|}{$2005-2010$} & \multicolumn{2}{|c|}{$2010-2015$} & \multicolumn{2}{|c|}{$1990-2015$} \\
\hline Variáveis & SAR & SEM & SAR & SEM & SAR & SEM & SAR & SEM & SAR & SEM & SAR & SEM \\
\hline \multirow[t]{2}{*}{ A } & $-0,1462$ & $-0,0700$ & 0,1010 & 0.441 & $-0,0656$ & $-0,1393$ & 0,6012 & 0,6851 & 0,1077 & 0,0991 & 0,1918 & 0,1632 \\
\hline & $(0,103)$ & $(0,282)$ & $(0,083)$ & $(0,0000)$ & $(0,185)$ & $(0,027)$ & $(0,000)$ & $(0,000)$ & $(0,001)$ & $(0,002)$ & $(0,001)$ & $(0,000)$ \\
\hline \multirow[t]{2}{*}{ B } & $-0,4874$ & $-0,6197$ & $-0,4155$ & $-0,4761$ & $-0,7603$ & $-0,7788$ & 0,7951 & 0,8016 & $-0,5023$ & $-0,4905$ & $-0,8008$ & $-0,8136$ \\
\hline & $(0,000)$ & $(0,000)$ & $(0,000)$ & $(0,0000)$ & $(0,000)$ & $(0,000)$ & $(0,000)$ & $(0,000)$ & $(0,000)$ & $(0,000)$ & $(0,000)$ & $(0,000)$ \\
\hline \multirow[t]{2}{*}{$P$} & $-1,2175$ & & 0,7376 & & 0,1806 & & 0,1381 & & $-0,1600$ & & $-0,1935$ & \\
\hline & $(0.2596)$ & & $(0,0000)$ & & $(0,1026)$ & & $(0,2564)$ & & $(0,5315)$ & & $(0,4922)$ & \\
\hline \multirow[t]{2}{*}{$\Lambda$} & & 0,5770 & & 0,7039 & & 0,6167 & & 0,6531 & & 0,2461 & & 0,2612 \\
\hline & & $(0,0000)$ & & $(0,0000)$ & & $(0,0000)$ & & $(0,0000)$ & & $(0,0781)$ & & $(0,0323)$ \\
\hline \multicolumn{13}{|l|}{ Estatística } \\
\hline $\mathrm{AK}$ & \multicolumn{2}{|c|}{$\begin{array}{c}1,317 \\
(0,2510)\end{array}$} & \multicolumn{2}{|c|}{$\begin{array}{c}0,542 \\
(0,4615)\end{array}$} & \multicolumn{2}{|c|}{$\begin{array}{c}6,299 \\
(0,0121)\end{array}$} & \multicolumn{2}{|c|}{$\begin{array}{c}6,473 \\
(0,0110)\end{array}$} & \multicolumn{2}{|c|}{$\begin{array}{c}1,820 \\
(0,1773)\end{array}$} & \multicolumn{2}{|c|}{$\begin{array}{c}2,177 \\
(0,1401)\end{array}$} \\
\hline
\end{tabular}

Fonte: Elaboração própria.

Nota: $\mathrm{O}$ termo entre parênteses indica os erros-padrão.

Todos os modelos corroboram a hipótese do processo de convergência espacial, isto é, indicam que a produtividade do café nas microrregiões menos produtivas cresceu a uma taxa maior do que as microrregiões mais produtivas. Os resultados também ratificam que o modelo de erro-espacial seria o mais adequado dado que apresentou o coeficiente $\lambda$ significativo em todos os períodos relevantes, enquanto, no modelo SAR, o coeficiente $\rho$ não significativo em quase todos os subperíodos.

\section{Considerações finais}


O objetivo do presente artigo foi analisar a produtividade média do setor cafeeiro, a nível microrregional, da região sudeste no período de 1990, 1995, 2000, 2005, 2010 e 2015. Utilizou-se a Aede que corresponde às estatísticas globais e locais.

Foi possível verificar a distribuição geográfica da produtividade do café e concluir que a produção geográfica desse produto não se distribui de forma homogênea ao longo do período analisado. Em todos os anos e para todas as convenções analisadas, os valores foram positivos e significativos, indicando que existe autocorrelação espacial global, portanto as políticas públicas adotadas nessas regiões que apresentaram o cluster AA mostraram-se eficientes quanto ao desenvolvimento da cultura de café para a economia da região como um todo.

De modo geral, entre 2005 e 2015, as microrregiões do norte do estado de Minas Gerais compunham as regiões com elevada produtividade evidenciando os resultados dos maciços investimentos internos e externos na região. Por outro lado, o grupo de regiões com baixa produtividade é representado por algumas microrregiões da Mesorregião Sul Fluminense e Mesorregião Metropolitana do Rio de Janeiro, bem como pelas microrregiões do litoral sul paulista.

$\mathrm{Na}$ estatística $I$ de Moran, houve aumento na dependência espacial entre as microrregiões em 1990 a 2015, ou seja, houve uma tendência de concentração espacial no que tange à produtividade do café. Localmente, as microrregiões com produtividade acima da média tendem a estar cercadas por microrregiões na mesma situação. Pode-se concluir que, em grande parte, tal fato se deve à estrutura produtiva adotada pelas microrregiões, pois na configuração dos clusters AA predominam as grandes produções cafeeiras, enquanto, nos clusters $\mathrm{BB}$, verificou-se o predomínio da policultura agrícola.

Foram testados os modelos de MQO, o SAR e o SEM. Para os subperíodos de 1990/1995, 2000/2005, 1990/2015, os Multiplicadores de Lagrange mostraram que a dependência espacial se manifesta tanto na defasagem como no erro. $\mathrm{O}$ valor da função no modelo de erro espacial é o mais indicado para explicar o processo de convergência absoluta da produtividade do café dado que o ML do erro é significativo nos subperíodos mencionados.

Esse tipo de estudo se mostra extremamente relevante na identificação de clusters de regiões produtoras da cafeicultura, contribuindo para melhor entendimento da distribuição espacial da produção. Assim é possível que os formuladores de políticas públicas identifiquem as regiões com um potencial latente para o desenvolvimento da produção de café. 


\section{Referências}

ALMEIDA, E. Econometria espacial aplicada. Alínea: Campinas, 2012.

ALMEIDA, E. S. de; PEROBELLI, F. S.; FERREIRA, P. G. C. Existe convergência espacial da produtividade agrícola no Brasil? Revista de Economia e Sociologia Rural. v. 46, n. 1, p. 31-52, 2008. DOI: https://doi.org/10.1590/S0103-20032008000100002_

ANSELIN, L. Spacial econometrics: methods and models. London: Kluwer Academic, 1988. DOI: https://doi.org/10.1007/978-94-015-7799-1

BRASIL. Ministério da Agricultura, Pecuária e Abastecimento. Culturas. Café. Saiba Mais. Disponível em: http://www.agricultura.gov.br/vegetal/culturas/cafe/saiba-mais. Acesso em: dez/2016.

BRASIL. Embrapa. Brasil produzirá 49 milhões de sacas de café em 2016. Disponível em: https://www,embrapa,br/busca-de-noticias/-/noticia/16659885/brasil-produzira49-milhoes-de-sacas-de-cafe-em-2016. Acesso em dez/2016.

Companhia Nacional de Abastecimento - CONAB. Acompanhamento da Safra Brasileira: Café. V.3, SAFRA 2016, N. 4. Quarto levantamento Dezembro 2016, Brasília: CONAB, 2016. Disponível em: http://www.conab.gov.br/OlalaCMS/uploads/arquivos/16 12_27 16 26 261 boletim cafe portugues - 40 lev - dez.pdf. Acesso em dez/2016.

CONSELHO NACIONAL DO CAFÉ. Café do Brasil - características agronômicas. 2017. Disponível em: http://www.cncafe.com.br/site/interna.php?id=26 . Acesso em maio/2017

DINIZ, S. S.; CÂMARA, M. R. G.da; MASSAMBANI, M. O.; ANHESINI, J. A. R.; SESSO FILHO, U. A. Análise espacial da produtividade da laranja dos municípios do estado de São Paulo: 2002-2010. In: CONGRESSO DA SOCIEDADE BRASILEIRA DE ECONOMIA, ADMINISTRAÇÃO E SOCIOLOGIA RURAL - SOBER. Anais... Vitória, julho de 2012.

DO VALE, A. R.; CALDERARO, R. A. P.; FAGUNDES, F. N. A cafeicultura em Minas Gerais: estudo comparativo entre as regiões Triângulo Mineiro/Alto Paranaíba e Sul/Sudoeste. Campo-Território: Revista de Geografia Agrária, v. 9, n. 18, 2014. 
FAVRO, J.; MARCONATO, M.; ALVES, A. F. Convergência da Produtividade do Milho no Brasil: Uma Análise Espacial. In: $54^{\circ}$ Congresso da Sociedade Brasileira de Economia, Administração e Sociologia Rural (SOBER). Anais... Maceió - AL, 2016. DOI: https://doi.org/10.1590/1234-56781806-94790540307

FOCHEZATTO, A.; STÜLP, V. Jose. Análise da convergência da produtividade da mão-de-obra agropecuária entre os estados brasileiros: aplicação de matrizes de Markov, 1990-2000. Rev. Econ. Sociol. Rural. v.46, n.3. Brasília julho/setembro 2008. DOI: https://doi.org/10.1590/S0103-20032008000300007

International Coffe Organization - OIC. Overview of the Global Coffee Trade. Infographics on the Global Coffee Trade \#CoffeeTradeStats. Disponível em: http://www.ico.org/coffee-trade-statistics-infographics.asp\#sthash.e6wvUytq.dpuf . Acesso em: dez/2016

JÚNIOR, J. N. de O., CASTELAR, I.; FERREIRA, R. T. Convergência microrregional no setor agrícola usando um modelo Threshold. In: Encontro Nacional de Economia. Anais... Recife, ANPEC, 2007.

LOPES, J. L. Avaliação do processo de convergência da produtividade da terra na agricultura brasileira no período 1960 a 2001, 2004. Tese (Doutorado em Ciências) Escola Superior de Agricultura Luiz de Queiroz, Universidade de São Paulo, Piracicaba. SP, 2004.

MINISTÉRIO DA AGRICULTURA, PECUÁRIA E ABASTECIMENTO. Café no Brasil. Disponivel em: http://www.agricultura.gov.br/assuntos/politica-agricola/cafe/cafeicultura-brasileira . Acesso em: nov./2017.

PEROBElLI, F., Salgueiro; FARIA, W. R.; FERREIRA, P. G. C. Análise da convergência espacial do PIB per capita no estado de Minas Gerais. Revista Brasileira de Estudos Regionais e Urbanos. v. 1, n. 1, p. 85-113, 2009.

PINHEIRO, M. A.; DA SILVA NETO, W. A.; PARRÉ, J. L. Um estudo exploratório sobre os efeitos espaciais na produtividade do milho no Paraná. In: ENCONTRO DE ECONOMIA PARANAENSE, Anais... Curitiba, ANPEC, 2007. 
TEIXEIRA, R. F. A. P.; BERTELLA, M. A. Distribuição Espaço-temporal da Produtividade Média do Café em Minas Gerais: 1997-2006. Análise Econômica. v. 33, n. 63, 2015. DOI: https://doi.org/10.22456/2176-5456.25814

SOUZA, R. M.; PEROBELLI, F. S. Diagnóstico espacial da concentração produtiva do café no Brasil, no período de 1991 a 2003. Revista de Economia e Agronegócio, v. 5, n. 3, p. 353-377, 2007.

VALE, S. M. L. R.; TEIXEIRA, M. B.; SANT'ANNA, J. C. O. Estilos gerenciais e eficácia administrativa na produção de café. Revista de Economia e Agronegócio, Viçosa, v. 4, n. 4, p. 533-552, 2006.

VERÍSSIMO, T. O. O capitalismo agrário na atividade cafeeira no município de Patrocínio (MG). Espaço em Revista, v. 17, n. 1, p. 85-102, 2015.

Recebido em 29.12.17

Aprovado em 09.01.19 